\section{THE USE OF GYROSTATS.}

$A \mathrm{~T}$ a recent meeting of the Physical Society a model was exhibited which purported to illustrate $\mathrm{Mr}$. Brennan's mono-rail railway. Prof. Perry, president of the society, made the following remarks, which he was afterwards requested to edit and publish :-

In 1874 two famous men made a great mistake in endeavouring to prevent the saloon of a vessel from rolling by using a rapidly rotating wheel. Mr. MacFarlane Gray pointed out the mistake. It is only when the wheel is allowed to precess that it can exercise a steadying effect; the torque or moment which it exerts is equal to the angular speed of precession multiplied by the moment of momentum of the spinning wheel.

It is astonishing how many engineers who know the laws of motion of translation are ignorant of angular motion, and yet the analogies between the two sets of laws are perfectly simple. I have set out these analogies in my book on "Applied Mechanics."

The last of these, between centripetal force on a body moving in a curved path and torque or couple on a body rotating about an axis, is the simple key to all gyrostatic and spinning-top calculations. When the spin of a top is greatly reduced, it is necessary to remember that the total moment of momentum is not about the spinning axis (see my "Applied Mechanics," p. 594). Correction for this is, I suppose, what introduces the complexity which scares the students of the subject of the vagaries of tops; but in all cases that are likely to come before an engineer, it would be absurd to study such a correction, and consequently calculation is exceedingly simple.

Inventors using gyrostats have succeeded in doing the following things:-

(r) Keeping the platform of a quickfiring gun level on board ship, however the ship may roll or pitch. Keeping a submarine vessel or flying machine with any plane exactly horizontal or inclined in any specified way. These were probably first described by Mr. Brennan. It is easy to effect such objects as these without the use of a gyrostat. By means of spirit levels it is possible to command powerful electric or other motors to keep anything always level. The actual methods employed by Mr. Beauchamp Tower (an hydraulic method) and by myself (an electric method) depend upon the use of a gyrostat which is really a pendulum, the spinning axis being vertical.

(2) Greatly reducing the rolling or pitching of a ship, or the rolling of a saloon in the ship. This is the problem which Mr. Schlick has solved with great success, at all events in the case of torpedo-boats.

(3) In Mr. Brennan's mono-rail railway, keeping the resultant force due to weight, wind pressure, centrifugal force, \&c., exactly in line with the rail, so that however the load on a waggon may alter in position, and although the waggon may be going round a curve, the waggon is quickly brought to a position such that there are no forces tending to alter its angular position. The car leans over towards a sudden gust of wind or towards the centre of curvature if going round a curved rail.

(4) I need not refer to such matters as the use of gyrostats in the correction of compasses on board ship.

Problems (2) and (3) are those to which I wish to refer. It is to be remembered that without gyrostatic apparatus a ship is necessarily stable, a mono-rail waggon is unstable.

Mr. Schlick uses a large wheel of ten or twenty tons revolving about an axis EF (Fig. I), the mean position of which is vertical. Its bearings are in a frame EFCD, which can move about a thwartship axes $C D$. Its centre of gravity is below this axis. Iset the ship have rolled through the small angle $R$ from its upright position; the axis EF has precessed through the angle $P$ from a vertical position. Let 0 stand for $d / d t$. Let the moment of NO. 2002, VOL. 77$]$ momentum of the wheel about its axis be $m$. Now if the ship were held fast so that she could not roll, we might study the vibratory motion $P$. The effect of the roll is merely to introduce a term $m \theta \mathrm{R}$ increasing $\mathrm{P}$. Thus we have

$$
\mathrm{I}_{1} \theta^{2} \mathrm{P}+f \theta \mathrm{P}-m \theta \mathrm{R}+b \mathrm{P}=0 \text {. . . . (I) }
$$

where $f \theta \mathrm{P}$ is a fluid friction introduced by dash pots acting at $A$ and $B, b P$ is the righting moment of the frame, and $I_{1}$ its moment of inertia about the thwartship axis. Now write out the usual equation of motion of the ship vibrating about a longitudinal axis through its centre of gravity, its moment of inertia being I, but introduce a moment $m \theta \mathrm{P}$ tending to diminish $\mathrm{R}$.

Then we have

$$
\mathrm{I} \theta^{2} \mathrm{R}+\mathrm{F} \theta \mathrm{R}+m \boldsymbol{\theta} \mathrm{P}+a(\mathrm{R}-\boldsymbol{\alpha})=0 .
$$

if $\alpha=\alpha_{0} \sin q t$ is the thwartship inclination of the sea to the horizontal, and $a$ is the righting moment of the ship per unit angle, being the weight of the ship multiplied by the metacentric height. $\mathrm{F} \theta \mathrm{R}$ is the moment due to friction against the sea.

Solving these equations just as if $\theta$ were a constant, we have from (I)

so that (2) becomes

$$
\mathrm{P}=\frac{m \theta \mathrm{R}}{\mathrm{I}_{1} \theta^{2}+\jmath \theta+b},
$$

$$
\left(\mathrm{I} \theta^{2}+\mathrm{F} \theta+a+\frac{m^{2} \theta^{2}}{\mathrm{I}_{1} \theta^{2}+f \theta+b}\right) \mathrm{R}=a \alpha . . .
$$

Clearing of fractions we find

$\left\{\mathrm{II}_{1} \theta^{4}+\left(\mathrm{FI}_{1}+f \mathrm{I}\right) \theta^{3}+\left(a \mathrm{I}_{1}+b \mathrm{I}+m^{2}+\mathbf{F} f\right) \theta^{2}+(b \mathrm{~F}+a f) \theta+a b\right\} \mathrm{R}=$ $\left(\mathrm{I}_{1} \theta^{2}+f \theta+b\right) a a$. . . (4)

Replacing $\theta^{2}$ by $-q^{2}$, and $\theta^{4}$ by $q^{4}$ (see my "Calculus for Engineers," p. 237), we can at once express $\frac{R_{0}}{\alpha_{0}}$ if $R_{0}$ is the amplitude of the roll, and, of course,

$$
\mathrm{P}_{0}=\frac{m q \mathrm{R}_{0}}{\sqrt{\left(b-\mathrm{I}_{1} q^{2}\right)^{2}+f^{2} q^{2}}} \text {. }
$$

I am here studying the forced vibrations, and not the natural vibrations. In any particular case it is quite easy to calculate $R_{0} / \alpha_{0}$ for a number of values of $q$, and information is obtainable which is quite different from what comes from a study of the natural vibrations (that is, taking $a=0$ ). Besides, it is the very easiest kind of arithmetical calculation, replacing rather troublesome mathematics. To many, indeed I may say to all students, the calculation of the unreal roots of a biquadratic is troublesome, and this must be done if the natural vibration is to be studied. It is obvious that the real parts of the roots of the resulting equation in $R$ (when $\alpha$ is 0 ) are negative, and therefore the motion is stable. ${ }^{1}$

If, however, we make $a$ of (2) negative, as it is in the

1 The well known conditions that the real parts of all the roots of $\theta^{4}+a \theta^{3}$ $+b \theta^{2}+c \theta+d=0$ shall be negative, are that $a, b, c$, and $d$ shall be positive, and also that $a b c-c^{2}-a^{2} d$ shall be positive. 
Brennan case, is will be found that the motion is not stable. Even without friction the vibration would become greater and greater, and friction makes matters worse. Indeed, no form of the Schlick method can be applied to the Brennan waggon. But to return to the ship.

It will be found that the amplitude of $\mathrm{P}$ is much greater than that of $R$, and in practice it is necessary to have stops to prevent $\mathrm{P}$ becoming too great. Of course, when further increase of $\mathrm{P}$ is prevented by a stop, the roll proceeds as if the wheel were not spinning.

I have not seen it mentioned, but I should think that Mr. Schlick would let his wheel revolve like Mr. Brennan's, in a very perfect vacuum inside a case, because the power wasted in friction of a wheel against an atmosphere is proportional to the density of the atmosphere. I have found that the best shape of wheel is one like a fly-wheel with a thin disc inside the rim instead of arms; there is more moment of momentum per pound to be obtained in this way than by building up a wheel like a compound disc, as a gun is built up of tubes shrunk on; and also it is much better than the form of wheel adopted by Laval in his turbine. I need not say, also, that the moment of momentum per pound of steel is proportional to the radius of the wheel; the greater the radius, therefore, the better.

It is assumed that by the use of bilge keels and rolling chambers, and as low a metacentric height as is allowable, we have already lengthened the time of vibration and damped the roll $R$ as much as possible. Using (2) or (5), we find $P$ if $R$ is known, and usually the quick vibration is much more magnified in $\mathrm{P}$ than the slow one.

Let us consider a numerical example. Using engineers' units, let $\mathrm{I}=1 \mathrm{x}^{8}, \quad a=2 \times 10^{7}, \quad \mathrm{I}_{1}=7000, \quad b=70,000$ $m=2.5 \times 10^{6}$, and $\mathrm{F}=4 \times 10^{6}$, and let us find answers to (4) for two values of $f$. These numbers are nearly right for a vessel of 6000 tons, metacentric height 18 inches, with a natural period of fourteen seconds. Its gyrostat wheel weighs about ten tons, with a 6 -feet radius and an angular velocity of 100 radians per second. The frame and wheel have an oscillating period about the axis CD of about two seconds. These answers are compared with the case of the gyrostat not in action, that is, $m=0$, or precession prevented.

If the gyrostat is not in action, it is easy to sce that

$$
\mathrm{R}_{0}=\alpha_{0} \div \sqrt{\left(\mathrm{I}-\frac{\mathrm{I}}{a} q^{2}\right)^{2}+\frac{\mathrm{F}^{2} q^{2}}{a^{2}}}
$$

The natural vibration has a damping term $\Sigma^{-\mathrm{F} t / 2 \mathrm{I}}$ with a periodic time nearly $2 \pi, \frac{\mathrm{I}}{a}$.

I take $F$ such that the amplitude diminishes by about 25 per cent. in one period (about fourteen seconds). It will be noticed that the $F$ term of the formula (6) is important only near the critical $q$ or $q=0.4472$. It will be found that the F term in (4) is of insignificant effect.

Again, the values of $\frac{K_{0}}{\alpha_{0}}$ get large for large values of $q$, because there is a quick natural vibration as well as a slow one. I have not thought it worth while to tabulate these higher values.

\begin{tabular}{|c|c|c|c|c|}
\hline \multirow[b]{3}{*}{$\mathrm{T}$} & \multirow[b]{3}{*}{$q$} & \multicolumn{3}{|c|}{ Values of $\mathrm{R}_{0} / \alpha_{0}$} \\
\hline & & \multirow{2}{*}{$\frac{\begin{array}{c}\text { Gyrostat not } \\
\text { acting }\end{array}}{f={ }_{5} \times 10^{4}}$} & \multicolumn{2}{|c|}{ Ciyrostat acting } \\
\hline & & & $f=5 \times 10^{4}$ & $f=15 \times 10^{4}$ \\
\hline 630 & O.OI & $I^{\circ} \mathrm{O}$ & $1 \cdot 0$ & $I \cdot O$ \\
\hline 63 & 0.1 & $1 \cdot 05$ & $x \cdot I$ & $I \cdot I$ \\
\hline $3 \times 5$ & 0.2 & $1 \cdot 25$ & $I 6 I$ & $\mathrm{I} \cdot 32$ \\
\hline 21 & 0.3 & I 82 & 5.57 & $2 \cdot 77$ \\
\hline 157 & $\mathrm{C}_{4}$ & $4 \times 64$ & I.96 & 175 \\
\hline 14 & 0.4472 & II 19 & & \\
\hline 12.6 & 0.5 & 371 & 0.76 & 0.73 \\
\hline $\mathrm{II}_{4} 42$ & 0.55 & 1.92 & & \\
\hline $10 \%$ & 0.6 & $1 \cdot 25$ & 0.55 & $0.35^{1}$ \\
\hline
\end{tabular}

NO. 2002, VOL. 77 ]
It is interesting to calculate $\mathrm{P}_{0} \div \alpha_{0}$ for all values of $q$, and especially for the larger values of $q$.

Free Vibration.-Using the above numbers and $\alpha=0$, so that the ship is gradually coming to rest, we are led to

$$
\mathrm{R}=\mathrm{Ae}^{-0.02 t} \sin 0.447 t \text {. . . . / }
$$

if the gyrostat is not acting. This is a periodic time of fourteen seconds, and the damping is such as to reduce the amplitucle of roll by 25 per cent. in each complete period.

When the gyrostat is acting and $f=50,000$, we are led

$$
\mathrm{R}=\mathrm{A} \epsilon^{-0.025 t} \sin 0.324 t+\mathrm{B} \epsilon^{-3.573 t} \sin (2.519 t+e) .
$$

We may neglect the quick vibrations of $2 \frac{1}{2}$ seconds' period, which are damped out very" rapidly. The slower have a period of nineteen seconds, the amplitude of roll being diminished by 30 per cent. in every complete period. Nore that $\mathrm{P}_{0}=50 \mathrm{R}_{0}$ if $q=2.519$, and $\mathrm{P}_{0}=11 \cdot 3 \mathrm{R}_{0}$ if $q=0.324$.

When the gyrostat is acting and $f=3 \times 10^{5}$, or six times as great, we are led to

$$
\mathrm{R}=\mathrm{A \epsilon}^{-0.02 t}+\mathrm{B \epsilon}^{-42.2 t}+\mathrm{Ce}^{-0.201 t} \sin \mathrm{I}^{-} 703 t^{\prime} \text {, }
$$

so that the slower periodic motion has disappeared, and the quick one, the period of which is nearly $3 \cdot 7$ seconds, is rapidly destroyed. For both (8) and (9) it is interesting and easy to calculate $P$.

In solving the biquadratics which lead to such answers, let it be noticed that we are led usually to roots $-\alpha \pm \beta i$ and $-m \pm n i$, where $i=\sqrt{-1}, n$ and $m$ being much smaller than $\alpha$ and $\beta$. If we leave out the last two terms of

$$
\theta^{4}+a \theta^{3}+b \theta^{2}+c \theta+d=0 \quad \text {. . . . (10) }
$$

we get the larger roots, approximately; if we leave out the first two terms we may not get $m$, but we get a good approximation to $n$, and it is $n$ which it is most important to know. The following is a quick method of finding the roots with any amount of accuracy that is required. We know that

$$
\begin{aligned}
& a=2(\alpha+m), \\
& b=\alpha^{2}+\beta^{2}+m^{2}+n^{2}+4 \alpha m, \\
& c=2 m\left(\alpha^{2}+\beta^{2}\right)+2 \alpha\left(m^{2}+n^{2}\right), \\
& d=\left(\alpha^{2}+\beta^{2}\right)\left(m^{2}+n^{2}\right) .
\end{aligned}
$$

The numerical example given above, where $f=5 \times 10^{4}$, requires us to solve

$$
\theta^{4}+7 \cdot 16 \theta^{3}+1942 \theta^{2}+1 \cdot 83^{\theta}+2=0 .
$$

First assume that $m=0$, so that $\alpha=3.58$., We see then that the sum of $\alpha^{2}+\beta^{2}$ and $m^{2}+n^{2}$ is $19 \cdot 4^{2}$, and their product is 2 , so that we can find them.

$$
\begin{gathered}
x^{2}+19.42 x+2=0 \\
\text { give: } a^{2}+\beta^{2}=19.42, m^{2}+n^{2}=0.103 . \\
\text { Then } 0.915 \text { or } \frac{1}{2} c=19.42 m+3.58 \times 0.103 \\
\text { or } m=0.0282 .
\end{gathered}
$$

Secondly assume that $m=0.0282$, so that $\alpha=3.55 \mathrm{rg}$; taking $x^{2}+19 \cdot 02 x+2=0$ we siget $\alpha^{2}+\beta^{2}=19.02 ; m^{2}+n^{2}=0.10515$, $\frac{1}{2} c=0.915=19.02 m+3.553 \times 0.105$ gives $m=0.0285$.

Assuming $m$ to have this value, we mav proceed to a third calculation. In this way we get closer and closer to the true value of $m$, and therefore to the true values of $\alpha, \beta$, and $n$. In practice I find that the two calculations such as $I$ give here are sufficient.

It may be taken as roughly true from (4) that the effective moment of inertia of the ship is increased from I to $I+\frac{m^{2}}{b^{2}}$, so that the time of a slow vibration is multiplied by

$$
\left(\mathrm{I}+m^{2} / \mathrm{l} b\right)^{t} \text {. }
$$

If all ships and their gear are similar, it will be found that $m^{2} / \mathrm{I} b$ is inversely proportional to the dimensions. Thus if a roo-ton boat has its period increased by 50 per cent., then a perfectly similar ship of 2700 tons will have its period lengthened by only ig per cent.

It may be, however, that the proportions should be different in vessels of different size, and it is not fair without further experience to make a comparison which seems so unfavourable to the method. Besides, experience alone can show how the dash-pot friction may depend 
upon the size of the ship. Probably, too, large ships may be regarded as being steady enough already.

If we apply the Schlick method to Mr. Brennan's car, as $a$ of (4) (putting $\alpha=0$ ) is negative, there is instability of motion whether there is or is not friction. We may,

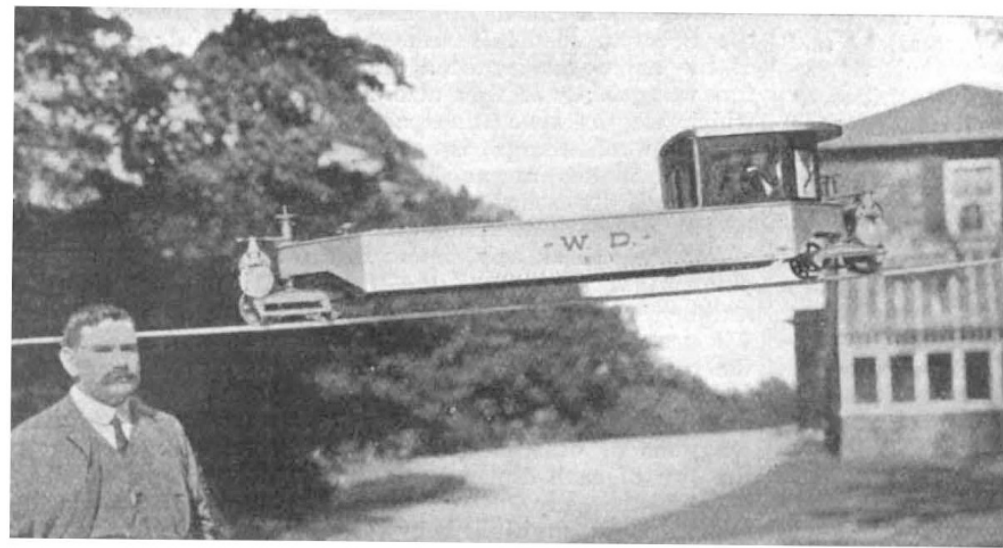

$F_{16} \cdot 2$.

however, do as the exhibitor of the model (at the Physical Society meeting) has done-make $b$ also negative. That is, make the gyrostat frame unstable by having the centre of gravity of the frame EAB above the axis DG. In this case, if there is absolutely no friction either of the $f$ or $\mathrm{F}$ kind, there will be steady vibrations about a mean position, but any friction will cause the swings to get larger and larger. It is to be noticed that even without friction there will be instability if $m$, the moment of momentum of the fly-wheel, is less than a certain amount. Mr. Brennan's method of working is quite different. Fig. 2 shows his model car (about 6 feet long). It is driven by electric accumulators carried by the car. His gyrostat wheels are driven by electromotors, not shown in Fig. 3 ; as they are revolving in nearly vacuous spaces they consume but little power, and even if the current were stopped they would continue running at a sufficiently high speed to be effective for a length of time.

It will be found that energy is wasted by friction, and also work has to be done in bringing the car to a new position of equilibrium, and all this is supplied by the electromotors. Should the gyrostat really stop or reach a certain low speed, two supports are automatically dropped, one on either side of the car; each of them drops until it reaches the ground, one of them dropping perhaps much farther than the other.

The real full-size car which $\mathrm{Mr}$. Brennan is now constructing may be pulled with other cars by any kind of focomotive, using electricity or steam or petrol, or each of its wheels may be a driving wheel. He would prefer to generate electric power on his train, and to drive every wheel with an electromotor. His wheels are so independent of one another that they can take very sharp curves and vertical inequalities of the rail. The rail is fastened to sleepers lying on ground that may have sidelong slope. The model car runs on an iron gas-pipe; the ground is nowhere levelled or cut, and at one place the rail is a steel wire rope spanning a gorge (Fig. 2). It is interesting to stop the car in the middle of this rope and to swing the rope sidewise, watching the perfect automatic balancing. The car may with confidence be left here for hours, balancing itself with nobody in charge. If the load on the car-great lead weights-be dumped about into new positions, the car effects balance with no apparent effort. But if, the car not running but merely balancing itself, a person standing on the ground pushes against it, the car will push in opposition, and by pushing judiciously a person can really disturb the car's vertical position considerably; it is as if an indignant animal were resisting the push. Left to itself now, the car quickly rights itself.

NO 2OO2, vOL. 77]
The car is supported by a mono-rail bogie at each end; each bogie has two wheels pivoted vertically and horizontally, so that curves may be very sharp and the ground may be uneven.

Fig. 3 is a diagrammatic representation of $\mathrm{Mr}$. Brennan's pair of gyrostats in sectional elevation and plan. The cases $G$ and $G^{\prime}$, inside which the wheels $\mathrm{F}$ and $\mathrm{F}^{\prime}$ are rotating in vacuo at the same speed and in opposite directions (driven by electromotors not shown in the figure), are pivoted about vertical axes EJ and $\mathrm{E}^{\prime} \mathrm{J}^{\prime}$. They are connected by spur-toothed segments $J J$ and $J^{\prime} J^{\prime}$, so that their precessional motions are equal and opposite. The whole system is pivoted about $\mathrm{C}$, a longitudinal axis. Thus when precessing so that $\mathrm{H}$ comes out of the paper, so will $\mathrm{H}^{\prime}$, and when $\mathrm{H}$ goes into the paper, so does $\mathrm{H}^{\prime}$.

When the car is in equilibrium the axes $\mathrm{KH}$ and $\mathrm{K}^{\prime} \mathrm{H}^{\prime}$ are in line $\mathrm{NN}^{\prime}$ across the car in the plane of the paper. They are also in a nearly horizontal line which is at right angles to the total resultant force on the car. I will call this the mid-position.

Let $\frac{1}{2} m$ be the moment of momentum of either wheel. Let us suppose the car to tilt so that the shelf $D$ comes up against $H$, the spinning axis (or a roller driven by

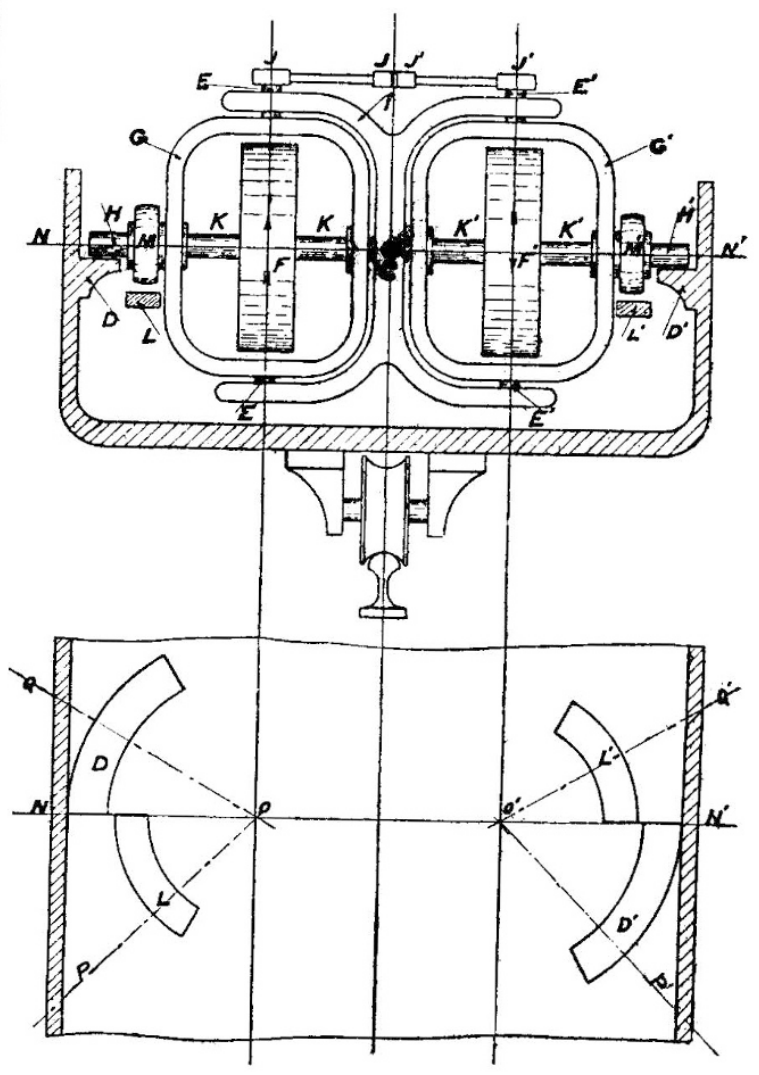

FiG. 3.

the spinning axis) of the gyrostat. $\mathrm{H}$ begins to roll away from me, and if no slipping occurred (but there is always slipping, and, indeed, slipping is a necessary condition) it would roll, that is, the gyrostats would precess with a 
constant angular velocity $\alpha$, exerting the moment $m a$ upon the shelf $D$, and therefore on the car. ${ }^{1}$

This precession continues until the roller and the shelf cease to touch. At first $\mathrm{H}$ lifts with the shelf, and afterwards the shelf moves downwards, followed for some distance by the roller. If the tilt had been in the opposite direction, the shelf $\mathrm{D}$ would have acted upon the roller $\mathrm{H}^{\prime}$, and caused just the opposite kind of precession, and a moment of the opposite kind.

We now have the spindles out of their mid-positions as $\mathrm{OQ}, \mathrm{O}^{\prime} \mathrm{Q}^{\prime}$. How are they brought back to $\mathrm{NOO}^{\prime} \mathrm{N}^{\prime}$ with $\mathrm{H}$ permanently lowered?

It is the essence of Mr. Brennan's invention that, after a restoring moment has been applied to the car, the spindles shall go back to the position $\mathrm{NOO}^{\prime} \mathrm{N}^{\prime}$ with $\mathrm{H}$ permanently lowered, so as to be ready to act again,

$\mathrm{He}$ effects this object in various ways. Some ways described in his patents are quite different from what is used on the model, and the method to be used on the fullsize waggon will again be quite different. I will describe one of these methods. Mr. Brennan tells me that he considers this old method to be crude, but he is naturally unwilling to allow me to publish his latest method.

$D^{\prime}$ is a circular shelf extending from the mid-position in my direction; $D$ is a similar shelf extending from the mid-position into the paper or away from me. It is on these shelves that $\mathrm{H}^{\prime}$ and $\mathrm{H}$ roll, causing precession, as I have just described. When $\mathrm{H}^{\prime}$ is inside the paper or when $\mathrm{H}$ is outside the paper they find no shelf to roll upon. There are, however, two other shelves, $\mathrm{L}$ and $\mathrm{L}^{\prime}$, for two other rollers, $M$ and $M^{\prime}$, which are attached to the frames concentric with the spindles. They are free to rotate, but are not rotated by the spindles. When they are pressed by their shelves $L$ or $L^{\prime}$ this causes negative precession, and they roll towards the $\mathrm{NOO}^{\prime} \mathrm{N}^{\prime}$ position. There is, of course, friction at their supports retarding their rotation, and therefore the precession. The important thing to remember is that $\mathrm{H}$ and $\mathrm{H}^{\prime}$ when they touch their shelves (when one is touching, the other is not touching) cause a precession away from the mid-position at a rate $\alpha$ which produces a restoring moment $m \alpha$ of constant amount (except for slipping), whereas when $\mathrm{M}$ or $\mathrm{M}^{\prime}$ touches its shelf $\mathrm{L}$ or $\mathrm{I}^{\prime}$ (when one is touching the other is not touching), the pressure on the shelf and friction determine the rate of precession towards the mid-position as well as the small vertical motion.

Suppose the tilt to be corrected is $R$, when $D$ presses $\mathrm{H}$ upward. The moment $m a$ and its time of action (the total momental impulse) are too great, and $R$ is overrcorrected; this causes the rollers $M^{\prime}$ to act on $L^{\prime}$, and the spindles return to the mid-position; they go beyond the mid-position, and now the roller $\mathrm{H}^{\prime}$ acts on $\mathrm{D}^{\prime}$, and there is a return to the mid-position and beyond it, and so it goes on-the swings of the gyrostats out of and into the mid-position, and the vibrations of the car about its position of equilibrium getting rapidly less and less, until again neither $\mathrm{H}$ or $\mathrm{H}^{\prime}$ nor $\mathrm{M}$ or $\mathrm{M}^{\prime}$ is touching a shelf. It is indeed marvellous to see how rapidly the swings decay. ${ }^{2}$

It wili be seen that by using the two gyrostats instead of one, when there is a curve on the line, although the plane $\mathrm{NOO}^{\prime} \mathrm{N}^{\prime}$ rotates, and we may say that the gyrostats precess, the tilting couples which they exercise are equal and opposite.

It is evident that this method of Mr. Brennan is altogether different in character from that of $\mathrm{Mr}$. Schlick.

1 I am supposing the precessional angles to be small ; when the angles are like $N O Q, N^{\prime} O^{\prime} Q^{\prime}$. Fig. 3 , the sum of the moments of the two gyrostats like $\mathrm{NOQ}, \mathrm{N}^{\prime} \mathrm{O}^{\prime} \mathrm{Q}$. Fig. 3 , the sum of the moments of the two gyrostats
would be ma cos $\mathrm{N} \cap Q, a$ being constant. if there were no slipping; but there is alwavs slipping, and the gond working of the apparatus requires
that there shall be such slipping. $a$ is not constant, and it is always less than that there shall be such slipping. a is not cons
what it wonld be if there was true rolling.

1 If in Fig. $3 \mathrm{R}$ is the angle which the waggon makes with its position of
and If in Fig. $3 \mathrm{R}$ is the angle which the waggon makes with its position of
equilibrium; if $M$ is the moment with which the shelf $D$ acts clockwise equilibrium; if $M$ is the moment with which the shelf $D$ acts clockwise
upon $H$, and $P$ is the angle of precession $Q \circ V_{\text {; }}$ ard if $\mu$ is the coefficient upon $\mathrm{H}$, and $\mathrm{P}$ is the angle of precession $\mathrm{Q} \circ \mathrm{V}$; and if $\mu$ is the coefficient
of friction between $\mathrm{D}$ and $\mathrm{H}$, then in the first part of the action above of friction hetween $\mathrm{D}$ and $\mathrm{H}$, than in the first part of the action above
described $\mathrm{I} \theta^{2} \mathrm{R}+m \theta \mathrm{P}-\mathrm{M}=0 . m \theta \mathrm{R}-i \theta^{2} \mathrm{P}+\mu \mathrm{M}=0,\left(\mathrm{I}_{1} \theta^{2}-h w\right) \mathrm{R}+\mathrm{M}=0$, if described $\mathrm{I} \theta^{2} \mathrm{R}+m \theta \mathrm{P}-\mathrm{M}=0$. $m \theta \mathrm{R}-i \theta^{2} \mathrm{P}+\mu \mathrm{M}=0,\left(\mathrm{I}_{1} \theta^{2}-h w\right) \mathrm{R}+\mathrm{M}=0$, if
$\mathrm{I}_{1}, \mathrm{I}$ and $\frac{1}{2} i$ are moments of inertia nf the waggnn about the rail, of the $\mathrm{I}_{1}, \mathrm{I}$ and $\frac{1}{2} i$ are moments of inertia nf the waggon abount the rail, of the
frames about $\mathrm{C}$, and of the frame $\mathrm{G}$ about $\mathrm{EJ}$; $\frac{1}{3} \boldsymbol{m}$ is the moment of

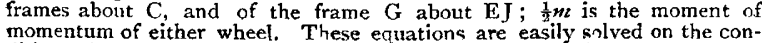
momentum of either wheel. These equations are easily solved on the con-
ditions that at $t=0, \mathrm{R}=\mathrm{R}, \theta, \theta \mathrm{R}=0, \mathrm{P}=0, \theta \mathrm{P}=0$. Assuming no play, that $\mathrm{s}$, that as soon as ' $\mathrm{H}$ leaves $\mathrm{D}, \mathrm{M}^{\prime}$ torches $\mathrm{L}$ ', we can now find the return to the mid-position from a new set of equations. Friction retards the return, it must be remembered. The motions are exceedingly interesting when numerical values of $\mathrm{T}_{1}, \mathrm{I}, i$, \&c., are taken, but the practical man will find $i$ more interesting to make an experimental study of what happens.
Work is here actually done which must be supplied by the electromotors. The restoring moment applied to the car may be made as great as we please by increasing the diameter of $H$. It is true that we cannot in this way alter the total momental impulse, and this is the important thing.

One of the most important things to know is this: the Brennan model is wonderfully successful; the weight of the apparatus is not a large fraction of the weight of the waggon; will this also be the case with a real car which weighs rooo times as much? If at any instant a condition of things is suddenly produced so that the waggon makes an angle $R_{0}$ with its position of equilibrium, if its weight is $W$, its centre of gravity at the height $h$ above the rail, if $I_{1}$ is its moment of inertia about the rail as axis, if $\frac{1}{2} m$ is the moment of momentum of each gyrostat wheel, the momental impulse $m \mathrm{P}_{1}$ ought, roughly, to be equal to $\mathrm{CR}_{0} \sqrt{\mathrm{I}_{1} \mathrm{~W}} h$, where $\mathrm{C}$ is a constant. $I$ use $P_{1}$ for the total angular precession at first. Now the tilt to be corrected, $\mathrm{R}_{0}$, may be due to wind pressure, to a sudden shifting of the centre of gravity, or to centrifugal force, and it is not easy to compare these things in waggons of different sizes. If, however, we take it that the size of each dimension of the waggon is multiplied by $n$ and the size of each dimension of the gyrostatic apparatus is multiplied by $p$, and the wheels have the same peripheral speeds, we find the following results :For wind, $R_{0}$ is proportional to $n^{-1}$. For centrifugal force it seems reasonable to take the speed of a waggon as proportional to $n$, and mean radius of rail curves proportional to $n^{2}$. In this case the result is again that $\mathrm{R}_{0}$ is proportional to $n^{-1}$. As for a possible accidental shifting of the centre of gravity because of the displacement of part of the cargo $w$ through the distance $d$, if we take $w \propto c n^{2}$ and $d<<n$, we find with greater and greater accuracy as $w$ is a smaller fraction of the whole weight, $\mathrm{R}_{0} \propto n^{-1}$.

Taking $P_{1}$, the maximum angle of precession, to be the same in all cases, the above relation leads to the result that $p=n^{\frac{7}{8}}$, or that the fractional weight of the apparatus as compared with that of the waggon is proportional to $W^{-0.125}$, where $W$ is the weight of the waggon. Thus, if we take $n=x 0$, that is, every dimension of the model multiplied by 10 or its weight by I0oo, then $p=7.5$, so that the weight of the gyrostat apparatus is only multiplied by 420 . If in the model the apparatus was io per cent. of the whole weight, in the large waggon the apparatus is only 4 per cent. of the whole weight. In fact, the larger the waggon the less proportion of its weight and volume is occupied by the a.oparatus, a result which must be very satisfactory to Mr. Brennan.

In the cases both of Mr. Schlick and Mr. Brennan, it has to be remembered that if the diameter of the wheel be increased in greater proportion than the dimensions of the ship or waggon, or other dimensions of the wheel, the proportional weight of the apparatus may be diminished. A wheel of twice the diameter, but of the same weight, may have twice the moment of momentum and may be twice as effective. I assume the stresses in the material to be the same.

\section{ON THE PHYSICAL ASPECT OF THE ATOMIC} THEORY. ${ }^{1}$

THE lecture began by setting out a physical reason a priori why matter should be constituted of discrete particles instead of being continuous. The requirements of physics demand an æther to serve as the means of communication between portions of matter out of contact with each other, and space can hardly be conceived as fully occupied simultaneously by two media, matter and æther; hence the matter must be constituted of discrete centres, or nuclei, determining permanent collocations of energy in the æether, which are, in fact, primordial atoms and their fields of force. The feasible problem of atomic physics is to build up an adequate idea of the dynamic constitution of these æthereal fields of force; there is the problem

1 Abstract of the Wilde Lecture of the Manchester Literary and Philo. sophical Society, delivered on March 3 by Prof. J. Larmor, Sec. R.S. 